COMECHINGONIA. REVISTA DE ARQUEOLOGÍA

Número 16, primer semestre 2012, pp. 167-184, Córdoba

ISSN 0326-7911

\title{
INDICIOS EN LA TIERRA: INHUMACIONES Y FOSFATOS. UNA EXPERIENCIA EN TUCUMÁN (ARGENTINA)
}

\author{
Paula V. Binder ${ }^{1}$ y Víctor Ataliva ${ }^{2}$ \\ ${ }^{1}$ INTI, Instituto Nacional de Tecnología Industrial. E-mail: paulitatuc@yahoo.com.ar \\ ${ }^{2}$ CAMIT, Colectivo de Arqueología, Memoria e Identidad de Tucumán. E-mail: victorataliva@yahoo.com.ar
}

Presentado el: 02/12/2011 - Aceptado 01/03/2012

\section{Resumen}

Se presentan los resultados de una intervención que se propuso generar una base teóricametodológica que respaldara una etapa pericial para la Justicia Federal de la provincia de Tucumán (Argentina). Durante la instancia de investigación confluyeron los aportes de la Ingeniería Industrial, de las Geociencias, de la Arqueología y la Química, posibilitando proponer un Plan de Trabajo que viabilice la detección de inhumaciones clandestinas en un territorio militar comprometido con las prácticas sociales genocidas llevadas a cabo en Tucumán desde mediados de la década de 1970. En este trabajo se discuten los resultados de una prospección geoquímica que permitió -a partir de la experimentación y evaluación en terreno y laboratorio- por un lado, contribuir con una propuesta de detección de inhumaciones clandestinas en contextos en los que se presume su existencia y, por otro, generar información para futuras intervenciones en el sitio histórico Estancia de San José de Lules (Tucumán).

Palabras claves: Prospección geoquímica, Prácticas sociales genocidas, Inhumaciones clandestinas, Tucumán.

\begin{abstract}
We present the results of an intervention that is proposed to generate a theoretical-methodological expert to back up a stage for a federal court in the province of Tucumán (Argentina). During the research came together the contributions of Industrial Engineering, Geoscience, Archaeology and Chemistry, making it possible a proposal that facilitate the detection of clandestine burials in a military territory committed to social practices genocidal carried out in Tucumán since the mid 1970's.In this paper we discuss the results of a geochemical survey -from a experimentation and evaluation in field and laboratory-on the one hand, to contribute to a proposal for detecting clandestine burials in contexts that are presumed to exist and, the other, to generate information for future interventions in the historical site of San José Estancia Lules (Tucumán).
\end{abstract}

Keywords: Geochemical survey, Genocidal social practices, Clandestine burials, Tucumán.

\section{Introducción}

Exponemos en este texto los resultados de una investigación cuyo objetivo fue generar una base teórica-metodológica para sustentar una etapa pericial para la Justicia Federal de Tucumán. En otras palabras, discutimos aquí las derivaciones de una investigación que 
viabilizó proponer un Plan de Trabajo que posibilite la detección de inhumaciones clandestinas en un territorio militar comprometido con las prácticas sociales genocidas (sensu Feierstein 2007) protagonizadas por las fuerzas armadas y de seguridad -y sus cómplices civiles- en Tucumán desde mediados de la década de 1970 hasta inicios de la siguiente. Este Plan tiene como base empírica una experiencia concreta: la intervención arqueológica y geoquímica efectuada en el sitio jesuita Estancia de San José de Lules, localizado en el pedemonte tucumano, a unos 16 kilómetros al sudoeste de la capital provincial. Asumíamos que dicha intervención en el sitio histórico nos permitiría capitalizar una experiencia a los fines de ajustar la técnica para ser puesta en práctica posteriormente (Binder 2008).

Ahora bien, más allá de la implementación de dicho Plan en esta causa judicial, nos proponemos discutir aquí los resultados de una instancia de prospección geoquímica que permitió: a) Generar información para futuras intervenciones en un sitio originalmente construido por la Compañía de Jesús (la Estancia); y, b) Contribuir con una propuesta de detección de inhumaciones clandestinas en contextos en los que se presume su existencia.

En el caso particular de Tucumán, el dispositivo desaparecedor requirió la conformación de paisajes de exterminio jalonados, fundamentalmente, por los espacios clandestinos de reclusión (los denominados Centros Clandestinos de Detención, CCD), pero también por aquellos lugares destinados a las inhumaciones tanto irregulares como clandestinas. Y precisamente éstas últimas fueron, hasta el hallazgo del denominado "Pozo de Vargas" en Tucumán (cf. Ataliva 2008), las evidencias de las prácticas sociales genocidas más escamoteadas para la Justicia, los organismos de Derechos Humanos y los equipos de investigación que abordan estas temáticas en Argentina. Aunque conllevaría una discusión extensa, ajena a los objetivos de esta contribución, es relevante señalar que los inconvenientes para la detección de este tipo de inhumaciones remitiría, entre otras circunstancias, a: la ausencia, inaccesibilidad o ambigüedad de los testimonios (de sobrevivientes, testigos o miembros de las fuerzas) que refieran a la localización de inhumaciones clandestinas; los intensos procesos post-depositacionales que, aunque restringidos a una escala temporal acotada (menos de cuatro décadas), alteraron las referencias materiales de los testigos (principalmente, por la intencionalidad de borrar toda evidencia superficial y subsuperficial del exterminio); que la mayoría de las intervenciones forenses estuvieron centradas en inhumaciones irregulares (esto es, en los cementerios). En este sentido, postular posibles herramientas de relevamiento y detección en contextos donde se presupone la existencia de inhumaciones clandestinas adquiere relevancia ante la diversidad de espacios empleados por las fuerzas del régimen para integrarlos al dispositivo desaparecedor.

La propuesta fue presentada a uno de los equipos de peritos ${ }^{1}$ que, al momento de llevar adelante la prospección geoquímica en la Estancia (2008), realizaba el relevamiento superficial en la ex Compañía de Arsenales Miguel de Azcuénaga² ${ }^{2}$ territorio militar en el que, según testimonios de sobrevivientes y ex miembros de las fuerzas de seguridad, funcionó un CCD en el que, además, se habrían realizado ejecuciones y la depositación de cuerpos en lo que comúnmente se denominan fosas (CONADEP 1984; Comisión Bicameral 1991) o inhumaciones clandestinas (sensu Somigliana y Olmo 2002). ${ }^{3}$ De estos testimonios se infiere el funcionamiento de un espacio de reclusión y la existencia de fosas de dimensiones variables (al menos 4 fosas y de unos $15 \mathrm{~m}^{2}$ de superficie).

A partir de la experimentación y evaluación en terreno y laboratorio, exponemos a continuación una instancia de intervención -la prospección geoquímica- que posibilitaría la 
detección de inhumaciones en el predio militar o en otros paisajes consustanciados con las prácticas sociales genocidas.

\section{Prospección geoquímica y análisis de fosfatos}

La prospección química ha permitido la detección de asentamientos arqueológicos y el despliegue de una serie de estudios sustentados en la premisa de que toda actividad humana genera alteraciones tanto físicas como químicas. En efecto, los análisis químicos para localizar / delimitar sitios y determinar "áreas de actividad", cuentan ya con más de medio siglo de aplicación en el ámbito de la Arqueología (Barba 1990). Cabe destacar que es extensa la producción científica en la que se exponen las investigaciones que involucran los análisis químicos en sitios arqueológicos (Barba et al. 1991; Díaz Vázquez 1999; Hardin et al. 1999; Terry et al. 2000; Barba y Domínguez-Bella 2003; entre otros). Del conjunto de herramientas químicas de prospección, el análisis de fosfatos constituye la técnica más empleada desde que Olof Arrhenius descubriera la correlación existente entre altas concentraciones de fosfatos y ocupaciones humanas (Barba 1990). Pero, ¿cuáles son los motivos por los que los fosfatos se presentan como indicadores de la presencia humana? Remitimos al ciclo del fósforo para responder esta pregunta.

Las actividades de las formaciones sociales generan cambios químicos que se materializan en el suelo. Tales modificaciones se manifiestan en anomalías en las concentraciones de carbono, calcio, nitrógeno, fósforo, con respecto a la media general de los suelos sin ocupaciones humanas (Woods, 1977, en: Eugenio y Murgo 1994:199). En el caso particular del fósforo, las investigaciones demostraron que es altamente estable, por lo que permanece depositado durante prolongados períodos. Cuando se descompone la materia orgánica se inicia la instancia de adición de fósforo al suelo en forma de fosfato, esto es, el fósforo de origen biológico (orgánico) forma fosfatos insolubles (minerales o fosfato inorgánico) (Barba et al. 1991; Díaz Vázquez 1999). Dado que artefactos y ecofactos contienen fósforo, como así también el propio ser humano (es decir, el tejido duro como los huesos y el tejido blando como la carne y la piel), éste se acumula y queda depositado (Barba et al. 1991). El fósforo orgánico ya depositado es transformado en inorgánico por microorganismos que lo mineralizan, al tiempo que disuelven el fósforo inorgánico emitiendo ácidos orgánicos y dióxido de carbono (Cook y Heizer 1965; Leonardi et al. 1999).

Debido a las características intrínsecas de los análisis de fosfatos (rápida extracción de muestras, bajo costo, la posibilidad de evaluar in situ los resultados), éstos permitieron abordar una diversidad de temáticas desde la Arqueología. Terry et al. (2000) distinguen tres ámbitos donde se desplegaron este tipo de análisis: a) Prospección; b) Estudios relacionados con las prácticas agrícolas y detección de áreas de agricultura; y c) Detección de "áreas de actividad" o, mejor dicho, para postular hipótesis sobre las posibles funciones que habrían cumplido ciertas localizaciones discretas hacia el interior de las unidades de vivienda $o$ entorno a ellas ( $v$. gr. sectores de preparación de alimentos, de acumulación de basura, de huertos familiares, cf. Parnell et al. 2002). En Argentina, por ejemplo, este tipo de análisis fue empleado en investigaciones que procuraban determinar los límites de un sitio (Eugenio y Murgo 1994), definir posibles actividades intrasitio (Ortiz 2003) y, en el caso de Tucumán, para abordar las actividades agrícolas prehispánicas en el Valle de Tafí (Sampietro Vattuone 2002; Roldán 2004, 2009; Roldán et al. 2008). 
En tanto antecedente específico de investigación que vincula el análisis de fosfatos con la detección de inhumaciones clandestinas, relevamos únicamente un caso en Sudamérica. Se trata de la intervención realizada en Vallegrande (Bolivia) a los fines de determinar el lugar de entierro de Ernesto Che Guevara y de los miembros de la columna insurgente cubano-boliviana bajo su mando. Como parte de la instancia de prospección, el equipo de investigación implementó técnicas geofísicas y geoquímicas. Con respecto a las segundas, se realizaron los análisis de $\mathrm{pH}$ y fosfatos de unas 1300 muestras. Aunque debido a una serie de inconvenientes (no vinculados con la técnica) la inhumación fue detectada a partir del trabajo con maquinaria pesada; una vez recuperados los restos óseos y materiales asociados, dicho equipo tomó 208 muestras del "suelo en la fosa a intervalos de 0,25 m en el área de $12 \mathrm{~m}^{2}$ que comprendia la misma. [Las muestras] fueron sometidas a análisis de fosfatos, $\mathrm{pH}$ y color de suelo. Esto permitió corroborar que potencialmente era posible localizar los restos empleando estas pruebas" (Rodríguez Suárez 2006:31-32).

Por otra parte, y relacionado con investigaciones sobre el pasado reciente en países de Latinoamérica (por ejemplo, en Guatemala, cf. Jacobo et al. 2004) o bien al ámbito de la criminalística (cf. Ritz et al. [eds.] 2009), la prospección geoquímica se constituye como una herramienta cada vez más empleada para el relevamiento de evidencias en contextos forenses.

Finalmente, es importante señalar que algunas de las investigaciones citadas conjugan distintas técnicas de prospección: entre las geofísicas, la resistividad eléctrica, el reconocimiento magnético y las ondas de radio; entre las geoquímicas, aquellas que se emplean para determinar restos inorgánicos (hierro, calcio, fosfatos, carbonatos, cloruros) y orgánicos (albúmina, ácidos grasos), y también para mensurar la alcanilidad y la acidez del suelo $(\mathrm{pH})$ (Barba et al. 1991). De hecho, la complementariedad de las distintas técnicas contribuiría a potenciar las posibilidades de detectar inhumaciones.

\section{Geoquímica: puesta a prueba en terreno y laboratorio}

Dado que las investigaciones realizadas en Tucumán no contemplaron el análisis de fosfatos como herramienta que posibilitara la detección de inhumaciones, se consideró relevante implementar la técnica y evaluarla en terreno y laboratorio para fundamentar, con mayor sustento empírico, la propuesta de intervención pericial en el predio militar. Para llevar adelante tal evaluación requeríamos de un sitio que: 1) Dispusiera de investigaciones previas y/o que actualmente sea objeto de estudio; 2) Se haya comprobado -o se presuma la existencia- de un sector con inhumaciones; 3) Las características pedológicas posibiliten -aunque en un primer momento tal consideración fuera apriorística- trazar correlaciones con el predio militar; y 3) Puedan tomarse muestras para ser analizadas.

El sitio histórico Estancia de San José de Lules (Departamento Lules, Tucumán), cumplía con todos los ítems enunciados, ya que: 1) Se realizaron intervenciones arqueológicas (cf. Anastasio et al. 2005 [1999]; Ataliva y López Campeny 2002; García Azcárate et al. 2002 [1999]) y se prevé la continuidad de las investigaciones; 2) Tales intervenciones permitieron detectar restos óseos humanos, pero no definieron espacialmente un sector del predio destinado a inhumaciones; 3) Aunque con características topográficas específicas, asumíamos, sino idénticos, al menos comparables procesos pedológicos en ambos sitios (la Estancia y el predio militar); y 4) Desde el Instituto de Arqueología y Museo (IAM, UNT), se dispuso de la autorización de Jorgelina García Azcárate (investigadora a cargo de proyectos que contemplan la Estancia) para la instancia de toma de muestras. 
La superficie actual del sitio es de unas 3 hectáreas ${ }^{4}$ y gran parte del conjunto arquitectónico que se observa en el presente, corresponde a la obra emprendida, hacia fines de siglo XIX, por el sacerdote dominico Ángel María Boisdron (figura 1). Anteriormente constituyó una estancia jesuita, pero ante la expulsión de la Compañía de Jesús (1767), la Junta de Temporalidades le concede esta propiedad a la Orden de los Dominicos (1781).

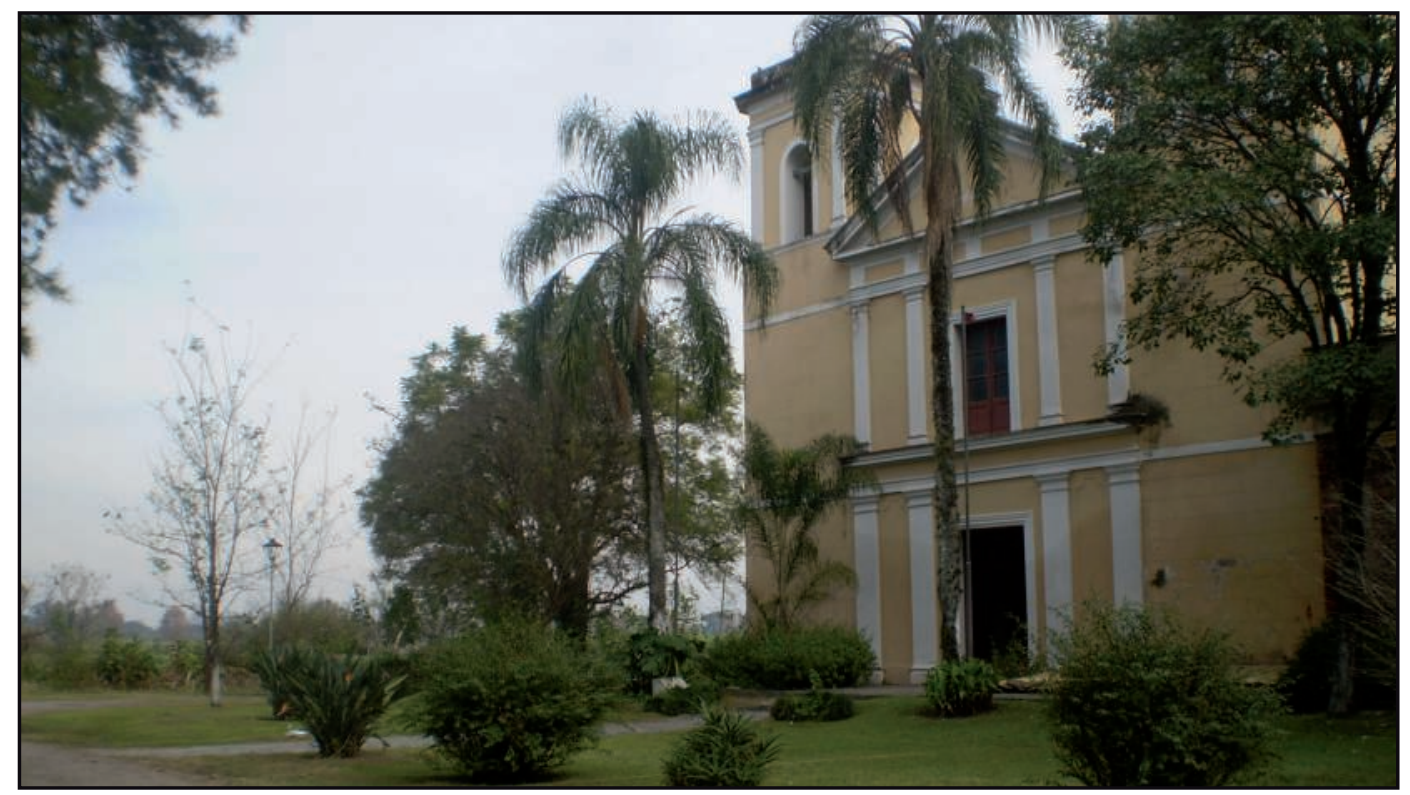

Figura 1. El "sector frente a la iglesia" (04/05/2008).

Durante el 2001 miembros del IAM realizaron una intervención de rescate a los fines de recuperar los restos óseos humanos que habían sido removidos de su posición original -en el sector definido con anterioridad como "frente a la iglesia" (sensu Anastasio et al. 2005 [1999])-, afectados por el empleo de una retroexcavadora que realizaba una zanja para el sistema de iluminación periférico del conjunto arquitectónico (proyecto a cargo de la Dirección Nacional de Arquitectura, Delegación Tucumán). Independientemente de la antigüedad de los restos óseos humanos (siglo XIX tardío o inicios del XX, cf. Ataliva 2010), cabe destacar que lo acotado de dicha intervención no posibilitó delimitar el espacio destinado a las inhumaciones (es decir, la superficie aproximada del sector empleado para depositar los cuerpos). Por otra parte, resultó relevante -a los fines de proyectar la profundidad para la extracción de las muestras- contar con información referida a dicha instancia de rescate (v. gr., las profundidades en las que se encontraban los restos óseos: hasta un metro desde la superficie actual).

A partir de las limitaciones económicas y de los objetivos de la intervención, se planificó el muestreo en función de dos hipótesis ( $\mathrm{H} 1$ y H2): H1, La disposición estratégica de los puntos de muestreo permitirá delimitar el sector destinado a los entierros humanos en el sitio histórico; $\mathrm{H} 2$, Los resultados reflejarán la depositación diferencial de fosfatos, alcanzando los valores máximos en el sector de inhumaciones y decreciendo a medida que nos distanciamos del mismo (Binder 2008). Con $\mathrm{H} 1$ se enfatizaba la relevancia de evaluar la localización de los puntos de muestreo, considerando la cantidad acotada de muestras posibles de analizar (un máximo de 15). Por otro lado, permitiría realizar un aporte a las investigaciones efectuadas en dicho sitio (esto es, 
delimitar el sector de inhumaciones). Con $\mathrm{H} 2$ se evaluaba la eficacia de la técnica y posibilitaba comprobar si la selección previamente planteada de los puntos de muestreo era acorde a lo enunciado (es decir: se manifestarían variaciones en la depositación de fosfatos).

Como primera instancia para la planificación del muestreo, evaluamos el sector de interés a partir de imágenes satelitales y fotografías aéreas; la revisión bibliográfica de los trabajos publicados e inéditos que abordan el sitio e inspecciones oculares (abril de 2008). Posteriormente, se planificó el muestreo considerando la superficie total del predio, el sector donde se detectaron las inhumaciones durante el 2001 y la cantidad de muestras factibles de analizar. Por ello la estrategia combinó el muestreo probabilístico con el no probabilístico. En el primer caso, se realizó un muestreo sistemático en el sector "frente a la iglesia"; en el segundo, se seleccionaron puntos de muestreo a determinadas distancias de dicho sector, sobre una transecta (transecta de referencia) que conectaba el "frente a la iglesia" con el punto de muestreo más alejado (en el ingreso al predio histórico).

Los pasos seguidos en gabinete y terreno, fueron: a) Con la imagen satelital se efectuó el reticulado del predio, asumiendo que con cuadrículas de 5 metros de lado se cubriría el sector de interés; b) Una vez reticulado el sector "frente a la iglesia" y los sectores centro y sur del predio, ubicamos los puntos de extracción de manera tal que permitiera, por un lado, abarcar totalmente el sector de inhumaciones (muestreo probabilístico) y, por otro, contar con muestras para confrontarlas con las del sector "frente"; y c) Localización de un punto de muestreo con el fin de evaluar el contenido natural de fosfato en este sector del pedemonte, considerando - para su ubicación- la posibilidad de que no haya sido un sector antrópicamente alterado.

El 4 de mayo de 2008 se realizó el muestreo. Como primera medida corroboramos la ubicación de los puntos de extracción y la posibilidad de tomar dos muestras más para delimitar con mayor precisión el sector de inhumaciones (puntos $\mathrm{A}^{\prime}$ e I'). En función de lo expuesto, detallamos el trabajo en el terreno:

a) Determinación de los puntos en el sector "frente a la iglesia", tomando como referencia la fachada de dicha construcción.

b) Una vez reticulado todo el "frente", quedó conformada una cuadrícula mayor de 20 metros de lado (con 16 cuadrículas en su interior, de $5 \times 5$ metros).

c) Se realizó la selección de los puntos ubicados de manera equidistante (puntos A; B; C; D; E; F; G; H; I; J).

d) Planteamos un punto de muestreo $(\mathrm{N})$, para determinar la depositación (natural) de fosfato en este sector del pedemonte tucumano.

e) Para delimitar con mayor precisión el sector de inhumaciones, planteamos dos puntos extras de muestreo $\left(\mathrm{A}^{\prime} ; \mathrm{I}^{\prime}\right)$.

f) Tomamos las muestras (dos por perforación), a un metro de profundidad, con motobarreno (figura 2).

g) Embolsamos e inventariamos las muestras para los análisis cuali y cuantitativos y su traslado a los respectivos laboratorios para la realización de los estudios.

Una vez realizada la toma de muestras, fueron trasladadas al Laboratorio de la Facultad de Ciencias Exactas (UNT), para el cuantitativo, y al Laboratorio de la Cátedra de Pedología (Facultad de Ciencias Naturales e Instituto M. Lillo, UNT), para el análisis cualitativo. ${ }^{5}$ Contar con estas determinaciones posibilitaría: a) Confrontar los resultados del análisis 


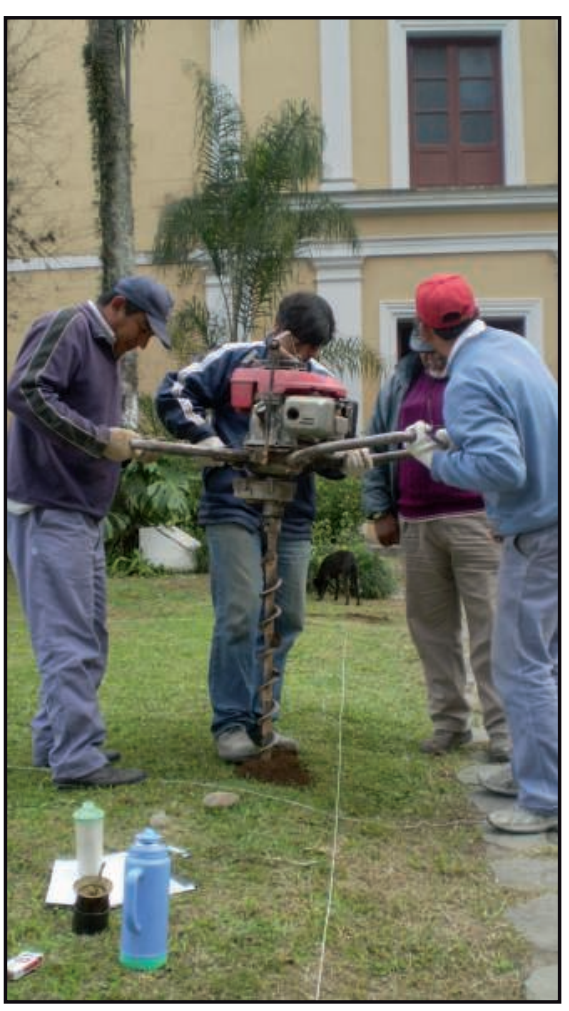

Figura 2. Realizando la perforación con motobarreno (extracción de la muestra F). cualitativo con el cuantitativo (tal cotejo permitiría determinar el grado de confiabilidad del análisis cualitativo y precisar qué escala de valores cuantitativos se ajustaban a la escala relativa resultante de la determinación cualitativa); b) Determinar estadísticamente la correlación entre ambas técnicas; y c) Evaluar comparativamente las limitaciones y ventajas de aplicar dichas técnicas.

El análisis cualitativo de las muestras se llevó a cabo con el "ensayo a la gota", técnica desarrollada por R. C. Eidt (Eugenio y Murgo 1996-98). Para optimizar la lectura, se realizó en tres oportunidades la determinación cualitativa. A diferencia de aquellas contribuciones que determinaron hasta cuatro valores nominales (por ejemplo, de 2 a 5 sensu Eugenio y Murgo 1996-98, o de 1 a 4 sensu Ortiz 2003), en los tres ensayos realizados establecimos una escala de 3 valores $(1,2,3)$; es decir, todas las muestras (y en las tres ocasiones), fueron reunidas en 3 grupos dependiendo de la intensidad/cobertura del azul y de la longitud de los rayos. Podríamos caracterizar, entonces, tales grupos como aquellos conformados por muestras con "bajo" (1), "mediano" (2) y "alto" (3) contenido de fosfato. También se determinó el $\mathrm{pH}$ de cada muestra con el fin de detectar variaciones y posibles relaciones con la depositación diferencial de fosfato. Con respecto al análisis cuantitativo, se realizó con el Método de Bray, recurrentemente empleado en el ámbito agro-químico para la determinación de fósforo en el suelo (Zalba et al. 2002; cf. Boschetti et al. 2003).

\section{Resultados}

En la tabla 1 confrontamos el análisis cuantitativo con la determinación cualitativa, el $\mathrm{pH}$ de cada muestra y la longitud de rayos. En la figura 3 se expone la disposición de los muestreos en la Estancia de San José de Lules y los valores resultantes del análisis cuantitativo (expresado en ppm o $\mathrm{mg} / \mathrm{kg}$ ).

En primera instancia, se advierte que los valores cuantitativos varían entre un mínimo de $20 \mathrm{mg} / \mathrm{kg}$ y un máximo de $235 \mathrm{mg} / \mathrm{kg}$. Ahora bien, la escala de valores cualitativos determinada en gabinete -después de sucesivos ensayos- concierne a los siguientes valores analíticos: un valor de 1 (“bajo”), corresponde a valores cuantitativos de 20 a $136 \mathrm{mg}$ / $\mathrm{kg}$; para 2 ("medio"), a valores entre 71 y $146 \mathrm{mg} / \mathrm{kg}$; finalmente, para 3 ("alto"), a valores cuantitativos entre 196 y $235 \mathrm{mg} / \mathrm{kg}$. Registramos, por lo tanto, una superposición de valores cuantitativos entre las escalas cualitativas de 1 y 2 . Sin embargo, se observa claramente que los valores cualitativos "altos" y de mayores longitudes de rayos quedan comprendidos en los máximos valores cuantitativos. Consecuentemente, al menos los "altos" valores de fosfato pueden ser registrados, sin ambigüedades, de manera cuali y cuantitativa. Al respecto, destacamos que la intensidad de la coloración azul y la longitud de rayos tienen su correlato 


\begin{tabular}{|c|c|c|c|c|}
\hline Muestra & $\begin{array}{c}\text { Determinación } \\
\text { Cuantitativa }(\mathrm{mg} / \mathrm{kg})\end{array}$ & $\begin{array}{c}\text { Escala } \\
\text { Cualitativa }\end{array}$ & $\begin{array}{c}\text { Longitud } \\
(\mathrm{cm})\end{array}$ & $p H$ \\
\hline M & 20 & 1 & 0,100 & 7,29 \\
\hline N & 54 & 1 & 0,050 & 7,17 \\
\hline L & 66 & 1 & 0,130 & 7,26 \\
\hline E & 132 & 1 & 0,150 & 7,28 \\
\hline K & 136 & 1 & 0,160 & 7,32 \\
\hline B & 71 & 2 & 0,500 & 6,66 \\
\hline A & 78 & 2 & 0,500 & 7,34 \\
\hline I' & 102 & 2 & 0,820 & 7,40 \\
\hline D & 121 & 2 & 0,520 & 7,14 \\
\hline A & 122 & 2 & 0,480 & 7,01 \\
\hline C & 130 & 2 & 0,500 & 6,95 \\
\hline F' & 146 & 3 & 0,740 & 7,12 \\
\hline J & 193 & 3 & 0,830 & 7,37 \\
\hline I & 197 & 3 & 0,760 & 7,45 \\
\hline H & 210 & 3 & 0,800 & 7,07 \\
\hline G & 216 & 3 & 0,750 & 7,45 \\
\hline F & 235 & &
\end{tabular}

Tabla 1. Determinaciones de laboratorio.

con los máximos valores cuantitativos. En este sentido, y para evaluar tal interpretación, se realizó el análisis de varianza, empleando los valores cualitativos y cuantitativos. ${ }^{6}$

Con respecto a los resultados estadísticos, el F calculado $(20,582)$ fue superior al $\mathrm{F}$ crítico $(3,74)$, de manera que la diferencia entre las medias de las variables cualitativas y cuantitativas es significativa, esto es, las variables son dependientes. ${ }^{7}$ Posteriormente, se calculó el coeficiente de correlación ( $\mathrm{r}$ ) entre las variables cuantitativas y cualitativas, lo que posibilitó determinar una correlación positiva significativa (figura 4).

Por otra parte, se confrontó la longitud de los rayos y los valores cuantitativos. En la figura 5 se aprecia el recorrido similar que describen ambas curvas, destacándose claramente dos aspectos: por un lado, a valores analíticos altos (a partir de los $193 \mathrm{mg} / \mathrm{kg}$ ), le corresponden las mediciones de rayos de mayor longitud (a partir de 0,740 cm); por otro, a valores cuantitativos más bajos (hasta $78 \mathrm{mg} / \mathrm{kg}$ ), le corresponden las menores longitudes (hasta $0,500 \mathrm{~cm}$ ). En otras palabras: los valores de alto contenido de fosfato se relacionan con los de mayor longitud de rayos; inversamente, los valores de bajo contenido con los de menor longitud de rayos. En este sentido, el $65 \%$ de los valores cuantitativos presentan una trayectoria similar, por lo que podríamos plantear que la confrontación entre valores cuantitativos y cualitativos adquiere mayor validez y consistencia cuando se consideran los extremos de la escala, es decir, es más factible la resolución interpretativa cuando se trata de confrontar los valores mínimos y máximos cualitativos con los valores analíticos mínimos y máximos, siendo de más compleja resolución los valores intermedios (en nuestro caso, aquellos valores relativos entorno a la escala "media" o 2). 


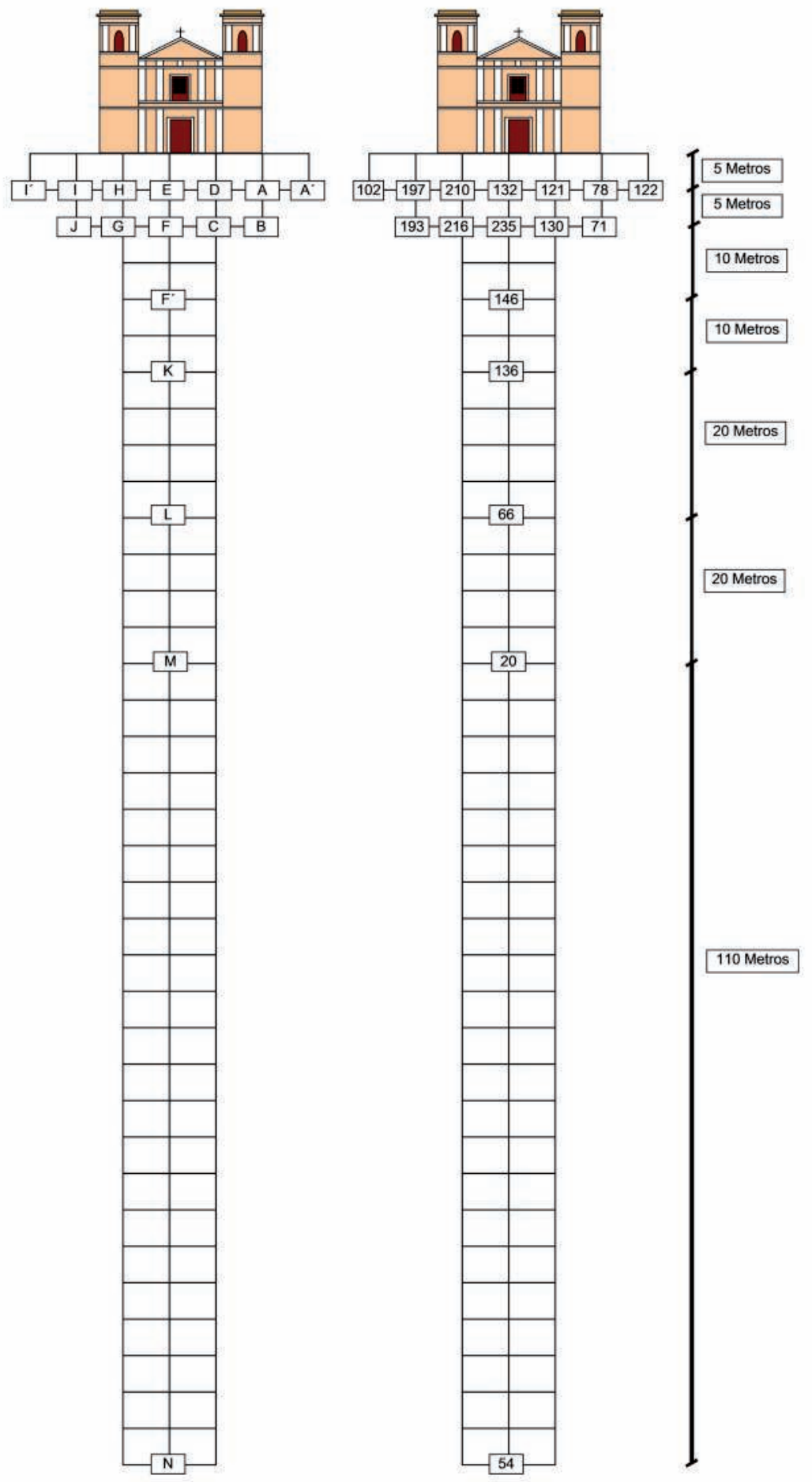

Figura 3. Esquema donde se aprecia la disposición respecto a la iglesia (vista de frente) de los puntos de muestreo (expuestos en planta). La transecta de referencia es la que culmina en $N$ (punto de muestreo que se encuentra a 180 metros de distancia desde la fachada de la iglesia). 


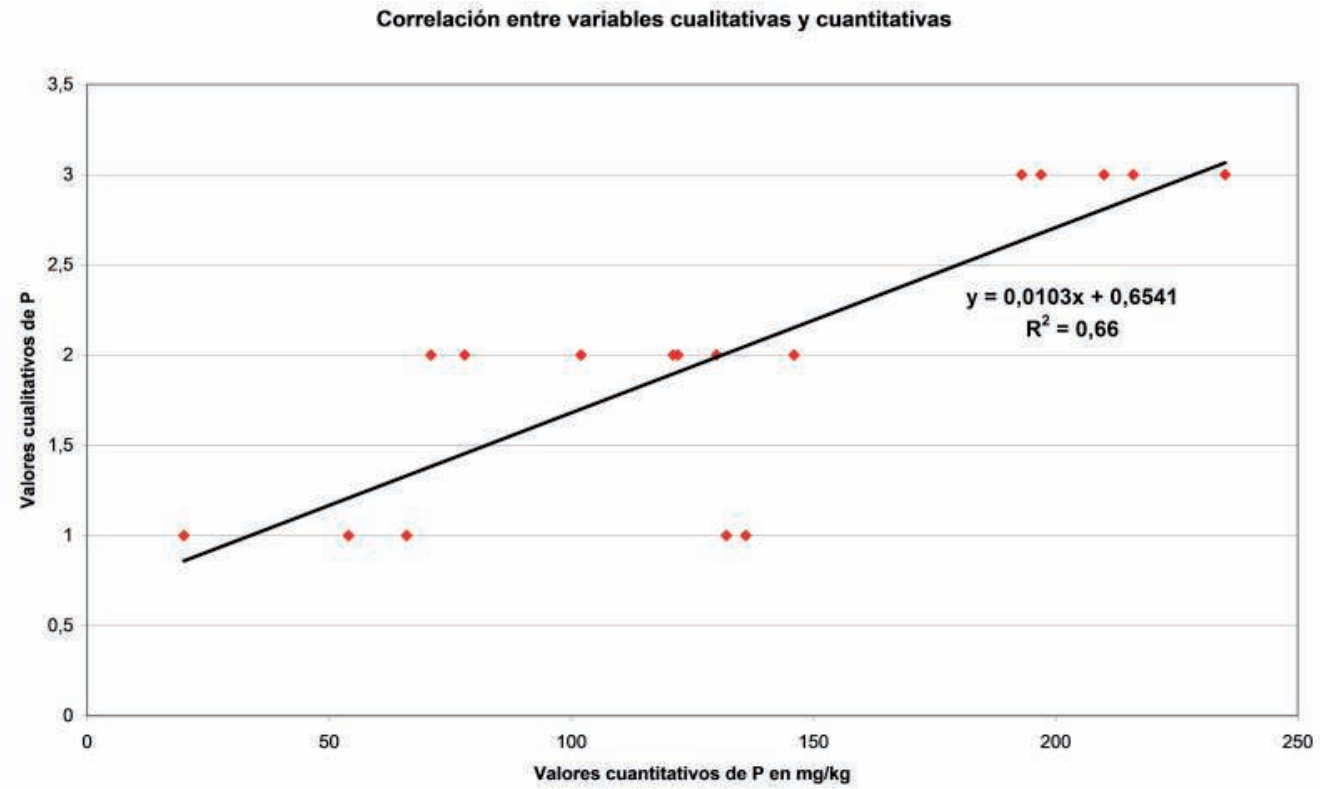

Figura 4. Coeficiente de correlación (r) entre variables cuantitativas y cualitativas.

Por último, y con el fin de evaluar la transecta con mayor cantidad de muestreos (transecta de referencia) -proyectada para comparar la distribución de fósforo en una línea imaginaria que uniera el punto de muestreo más alejado $(\mathrm{N})$ con el sector crítico ("frente a la iglesia")- en la figura 6 se exponen los valores analíticos que posibilitan vislumbrar las potencialidades del análisis cuantitativo. Como puede apreciarse, el valor más alto corresponde al sector donde

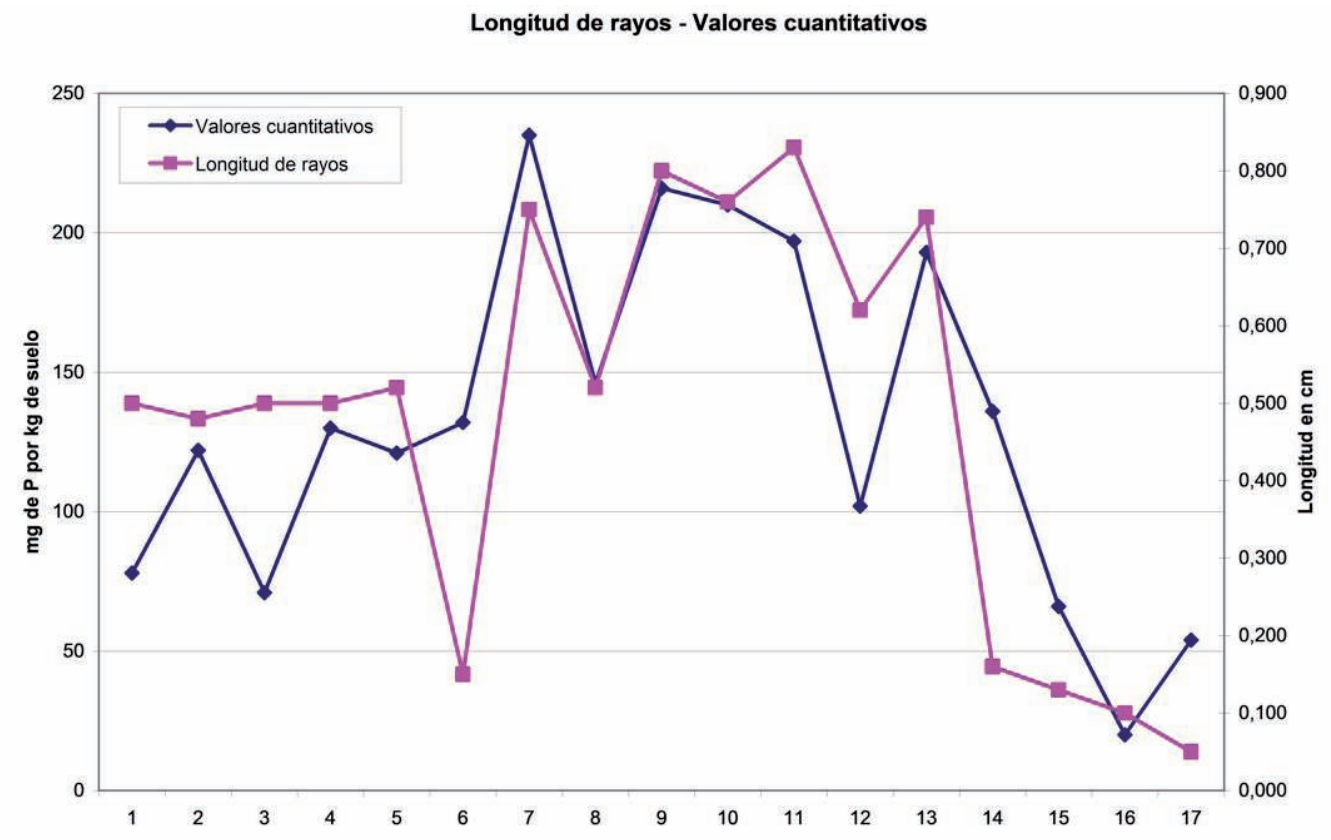

Figura 5. Comparación entre valores cuantitativos y longitud de rayos. 
efectivamente se recuperaron los restos óseos humanos durante la intervención de rescate de 2001. Independientemente del valor analítico que corresponde a E y que debemos evaluarlo desde una perspectiva cultural ${ }^{8}$, destacamos los valores decrecientes desde F a M. Esto es, a medida que nos alejamos de $\mathrm{F}$, disminuye la depositación de fósforo en la transecta, alcanzando el valor mínimo $(20 \mathrm{mg} / \mathrm{kg})$ en el punto M. A partir de las contribuciones históricas y arqueológicas se infiere que fueron recurrentes las inundaciones producidas por el río Lules (Ataliva y López Campeny 2002). Las mismas habrían afectado en numerosas ocasiones al predio y las instalaciones. Entonces, ¿es posible que el valor más bajo (muestra M) remita a una instancia de inundación que haya afectado la depositación natural de fosfato en este sector del predio? Es factible que estudios pedológicos específicos puedan contribuir a dar una respuesta. En todo caso nos interesa resaltar la coherencia de los resultados analíticos respecto a las hipótesis planteadas: a medida que nos alejamos del sector de inhumaciones disminuye la depositación de fósforo.

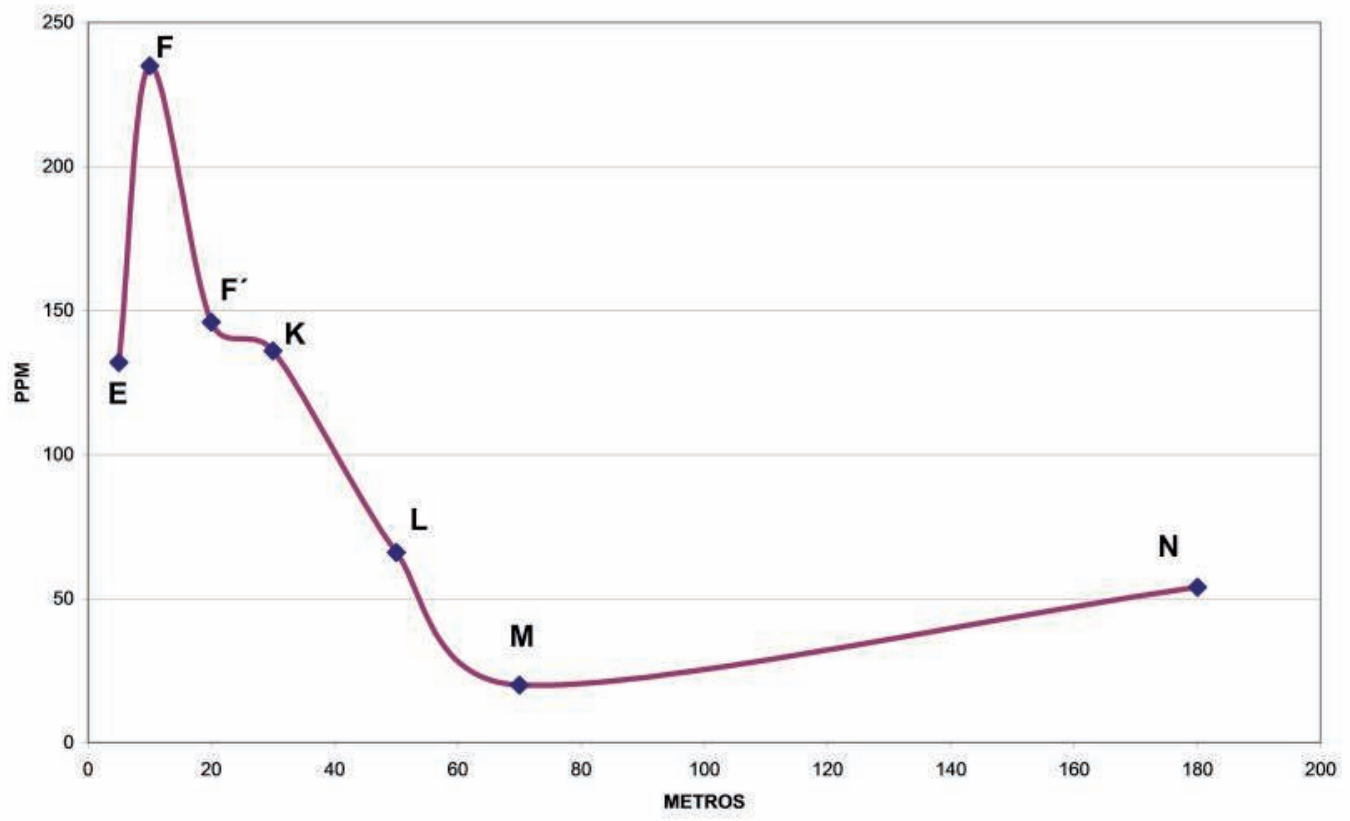

Figura 6. Valores cuantitativos en transecta de referencia.

Finalmente, y en función de los valores de $\mathrm{pH}$, resaltamos lo siguiente: la relevancia de detectar alguna relación entre $\mathrm{pH}$ y los valores analíticos radicaba en registrar anomalías en el pH que brindaran indicios de las inhumaciones, más allá de los valores analíticos. En este sentido, y como queda expresado en la tabla 1, no relevamos una relación directa entre los valores de $\mathrm{pH}$ y los cuantitativos.

\section{Discusión}

Conforme a los objetivos de la intervención en la Estancia, es decir, aportar críticamente a la evaluación de la prospección geoquímica en tanto vía de análisis susceptible de ser confrontada con otras técnicas que viabilicen la detección de inhumaciones en el predio militar ex Compañía de Arsenales (Binder 2008), exponemos a continuación las primeras consideraciones que resultaron del trabajo en terreno y gabinete. 
Destacamos, en un principio, que la instancia previa a la intervención en el terreno (análisis bibliográfico y de las imágenes satelitales, inspección ocular y prospección superficial), posibilitó plantear los puntos de muestreo y materializarlos posteriormente en el sitio. En este sentido, resaltamos la relevancia de haber planteado el muestreo teniendo como base la información generada por las intervenciones de rescate efectuadas durante 1998 y 2001. Es decir, al contar con información preexistente e imágenes aéreas, se pudo realizar la planificación seleccionando previamente los puntos de muestreo.

Con respecto a los resultados de los análisis cualitativos y cuantitativos, éstos reforzaron nuestras primeras percepciones acerca de la distribución diferencial de fosfato. De hecho, los análisis realizados permiten plantear que en la Estancia el sector destinado a los entierros humanos habría estado restringido al sector "frente a la iglesia". ${ }^{9}$ Tal como puede apreciarse en la figura 3 -y si bien el valor de $A^{\prime}$ no corresponde con lo esperado en función de las muestras A y B de la retícula ${ }^{10}$ - el sector "frente" comprendido por los puntos A, B e I, J (y probablemente hasta la muestra K), habría conformado el sector destinado a los entierros hacia -o desde- fines de siglo XIX o inicios del siguiente. Es decir, abarcaría una superficie aproximada a los $600 \mathrm{~m}^{2}$ (20 metros de frente por unos 30 metros desde la fachada hacia el ingreso al predio).

A partir de la experiencia con el motobarreno, interesa mencionar que éste impacta en el punto donde se realiza la extracción. Es decir, en este caso, implicó la extracción de una columna de tierra de 1 metro de altura por 2 pulgadas de diámetro $(5,08 \mathrm{~cm})$, por lo que el instrumental afecta el subsuelo de manera directa en el lugar puntual donde se realiza la extracción (consecuentemente, podría afectar -de hecho, lo hace- aquellas evidencias que se encuentren a lo largo de la columna).

A continuación se plantean una serie de consideraciones que, en el contexto judicial, podrían ser tenidas en cuenta para detectar las inhumaciones en el predio militar.

1) Para llevar adelante este tipo de intervenciones en un territorio militar es necesario relevar, analizar y sistematizar todo tipo de información referida a sus particularidades espaciales, topográficas, fitogeográficas, sociales, etc.; se debe disponer de documentación gráfica para diseñar el muestreo (fotografías aéreas, imágenes satelitales, etc.) y relevar los testimonios de sobrevivientes, ex miembros de las fuerzas de seguridad, entre otros, a los fines de confrontar y complementar la información que aportan las distintas fuentes. Tal instancia de intervención (junto a los resultados de la prospección superficial, sondeos exploratorios, etc.), posibilitará zonificar el predio según criterios de relevancia.

2) Como mencionamos al principio, de los testimonios se presume la existencia de un número no menor de 4 "fosas", de dimensiones variables y de una superficie aproximada a $\operatorname{los} 15 \mathrm{~m}^{2}$, estimadas en función de las declaraciones de dos ex miembros de Gendarmería Nacional (cf. CONADEP 1984; Comisión Bicameral 1991). A partir del volumen de tierra que conllevaría el cavado y posterior relleno de las mismas, es posible plantear que algunos rasgos (montículos, depresiones, etc.) que se releven en el predio podrían remitir a inhumaciones. Sin embargo, las intensas alteraciones posteriores (principalmente -léase: intencionalmentelas antrópicas) modificaron el aspecto superficial del territorio. En este sentido, los rasgos -y su entorno inmediato- podrían ser considerados como "puntos claves" o "críticos" para la extracción de muestras, aunque es recomendable que el tipo de muestreo a implementar considere tanto los rasgos como aquellos sectores del terreno que no los contengan (Binder 
2008). Por lo tanto, es altamente conveniente diseñar un muestreo probabilístico sistemático en las zonas definidas como de interés para la causa. Se debería tener presente el posible tamaño de las "fosas" para generar un espacio de muestreo donde los puntos de extracción dispuestos equidistantemente viabilicen la detección de anomalías en la depositación natural de sedimentos.

3) Según los testimonios, una vez arrojados el/los cuerpo/s, se depositaban sobre ellos leña, gomas, combustible, etc., y se procedía a la quema, por lo que algunas muestras de sedimento que se extraigan podrían ir acompañadas de cenizas, espículas de carbón, caucho, etc., aspecto sumamente importante ya que podrían dar indicios de las inhumaciones. En este sentido, el aporte de las geociencias es relevante dado que, incluso en terreno y a partir del análisis macroscópico de las muestras, se podría determinar preliminarmente (analizando las características granulométricas, de color, etc.) si se trata de sedimento depositado naturalmente o del producto de un relleno artificial (o de otra estructura de cavado: una trinchera, un lugar para arrojar basura, para detonar artefactos explosivos, etc.).

\section{Consideraciones finales}

A partir de la intervención llevada a cabo en la Estancia, es posible plantear, con algunas restricciones, la correspondencia existente entre los resultados cuantitativos y cualitativos. Dichas limitaciones se refieren a los valores "bajos" y "medios" de fosfato al confrontar la escala cualitativa con la analítica, por lo que la técnica cualitativa es muy confiable cuando registra valores "altos" de fosfatos. En función de los objetivos del equipo de peritos, dicha técnica posibilitaría relevar "altas" concentraciones, por lo que es apropiada su implementación para la detección de inhumaciones. Por otra parte, la determinación cualitativa (además de ser precisa con los valores "altos") es de aplicación sencilla, bajo costo, expeditiva y requiere de pocos operadores para la obtención de resultados, incluso a los minutos de ser extraída la muestra en el terreno.

Por último, y a partir de los valores de $\mathrm{pH}$ registrados en la Estancia y los posibles valores de $\mathrm{pH}$ que podrían arrojar las muestras en el predio militar, cabe mencionar lo siguiente: con un mínimo (de 6,66) y un máximo (de 7,45$)$ de valores que giran entorno al punto de neutralidad (7), la medición de $\mathrm{pH}$ no arrojó valores que posibiliten trazar relaciones directas con los valores cuantitativos. Permite postular, en todo caso, que los enterramientos humanos en determinados contextos no modificarían sustancialmente el $\mathrm{pH}$. Sin embargo, y como han demostrado distintas investigaciones, altos valores de $\mathrm{pH}$ (mayores de 8), necesariamente deben ser interpretados como producto de la actividad humana (Ortiz 2003). En este sentido, valores elevados de fosfato y un $\mathrm{pH}$ mayor de 8 darían indicios de un sector específico de una vivienda (por ejemplo, un fogón) destinado a los desechos y la quema (Barba 1990). Por otra parte, en dichos estudios se comprobó que la "distribución de pH en un piso sugiere la presencia de cenizas alrededor de los lugares de calentamiento, posiblemente anafes, y la trayectoria por donde se barrían las cenizas" (Barba et al. 1991:25). Por lo tanto, y en función de la dinámica de uso que aparentemente habrían tenido las "fosas", la determinación de pH también podría aportar a la detección de inhumaciones (o de anomalías en el suelo).

Los resultados de la prospección geoquímica vehiculizaron generar información para futuras intervenciones en la Estancia de San José de Lules, posibilitando prevenir otro impacto futuro en el sitio al delimitar el sector destinado a los entierros humanos. Por otro lado, los resultados de esta intervención viabilizaron generar una propuesta concreta para 
la detección de inhumaciones clandestinas en contextos de prácticas sociales genocidas en el ámbito local.

\section{Post scriptum}

Con posterioridad al envío del presente trabajo para su evaluación, se da a conocer públicamente el hallazgo de inhumaciones clandestinas en la ex Compañía de Arsenales Miguel de Azcuénaga (diciembre de 2011). Dado que la intervención pericial continúa vigente, la acotada reflexión que exponemos a continuación está fundada, principalmente, en consideraciones basadas en nuestra experiencia tanto en la Estancia (cf. Binder 2008; Binder y Ataliva 2009) como en el predio militar. ${ }^{11}$

Más allá de la técnica implementada para la reciente detección de las inhumaciones (empleo de maquinaria pesada para prospectar el subsuelo de un sector del predio), y dada la complejidad del territorio militar (por sus características topográficas -con marcadas pendientes, con sectores profundamente alterados por las prácticas militares comunes a todo distrito militar, por ejemplo, actividades de entrenamiento y detonación de explosivos, etc.por su extensión; por la intencionalidad de alterar y obturar las evidencias de las prácticas sociales genocidas; etc.), sostenemos que una intervención como la planteada aquí permitiría, en caso de que aún se encuentren inhumaciones sin registrar, la detección de éstas. De hecho, una forma de comprobar si la instancia geoquímica es viable podría ser, por ejemplo, efectuar el análisis de los arqueosedimentos asociados a los restos óseos recuperados en la ex Compañía, con lo cual se contaría objetivamente con parámetros comparativos que permitirían ajustar o descartar la técnica. En todo caso, la experiencia en la Estancia jesuita y el Plan de Trabajo oportunamente presentado (Binder 2008), permiten sostener que la prospección geoquímica se comporta como una herramienta alternativa para ser implementada en contextos donde se sospecha -o asume- la existencia de inhumaciones clandestinas.

\section{Agradecimientos}

A Nicolás Nieva, "Tero" Arancibia, Ruy Zurita, Alejandra García Araoz, Jorgelina García Azcárate, Patricia Cuenya, Lalo Valoy y equipo. Al CAMIT y al GIAAT. Finalmente, a los evaluadores del trabajo, quienes contribuyeron con ajustados y valiosos comentarios, los que fueron considerados en la versión final de este texto..

\footnotetext{
Notas

${ }^{1}$ Al Grupo Interdisciplinario de Arqueología y Antropología de Tucumán (GIAAT), Facultad de Ciencias Naturales e Instituto M. Lillo, Universidad Nacional de Tucumán.

${ }^{2}$ La superficie del predio es de, aproximadamente, 360 hectáreas y se encuentra a unos 7 kilómetros al norte de la capital tucumana (sobre Ruta Nacional Nº 9).

${ }^{3}$ Paralelamente a la intervención del GIAAT en el predio militar, también asumen como responsables de la pericia el Colectivo de Arqueología, Memoria e Identidad de Tucumán (CAMIT) y el Equipo Argentino de Antropología Forense (EAAF). El EAAF centró su intervención en la detección de las inhumaciones; por su parte, Ruy D. Zurita, M. Gloria Colaneri, Luciano R. Molina, Alejandro Leiva y Andrés S. Romano, miembros del CAMIT, abordaron de manera sistemática (durante el período comprendido entre 2010 y 2011), el interior del Galpón $N^{\circ}$ 9, aportando evidencias materiales que remiten al empleo de dicha instalación como el espacio de reclusión mencionado por los sobrevivientes de este CCD y los testimonios de ex miembros de Gendarmería Nacional.
} 
${ }^{4}$ Coordenadas del predio: ángulo NE, $26^{\circ} 54^{\prime} 25,72^{\prime \prime}$ de Latitud S y $65^{\circ} 19^{\prime} 24,43^{\prime \prime}$ de Longitud O; ángulo SE, $26^{\circ} 54^{\prime} 30,51^{\prime \prime}$ y $65^{\circ} 19^{\prime} 26,07^{\prime \prime}$; ángulo NO, $26^{\circ} 54^{\prime} 22,71^{\prime \prime}$ y $65^{\circ} 19^{\prime} 35,51^{\prime \prime}$; ángulo SO, $26^{\circ} 54^{\prime} 27,52^{\prime \prime}$ y $65^{\circ} 19^{\prime} 19,37^{\prime \prime}$.

${ }^{5}$ La determinación cualitativa que realizamos fue dirigida por la geóloga P. Cuenya, titular de la Cátedra de Pedología.

${ }^{6}$ Para ello se enunció, como Ho (Hipótesis nula), la ausencia de relación entre las variables cualitativas y cuantitativas (esto es, las primeras son independientes de las segundas); y como Ha (Hipótesis alternativa), la existencia de relación entre las variables cualitativas y cuantitativas (son variables dependientes).

${ }^{7}$ Por lo tanto, descartamos la Ho y aceptamos la Ha (es decir, existe relación entre los valores resultantes del análisis cualitativo y cuantitativo).

${ }^{8}$ Por ejemplo, podríamos plantear como hipótesis que el valor cuantitativo en este punto de muestreo estaría reflejando cierto comportamiento de los responsables de realizar las inhumaciones en la Estancia: al encontrarse en la vía de acceso más cercana y directa al interior de la iglesia, es posible que se haya procurado la menor remoción de tierra en dicho ingreso, y es por tal motivo que los valores cuantitativos de E y D reflejarían una depositación "media".

${ }^{9}$ Al menos el nivel de inhumaciones (hasta 1 metro de profundidad desde la superficie actual) que -dada las características de la presente intervención- registramos, correspondería a fines de siglo XIX e inicios del siguiente (Ataliva 2010). Por lo tanto, nuestras consideraciones están centradas en el período de ocupación correspondiente a los dominicos. Queda pendiente, entonces, determinar si se trata de un sector de inhumaciones con una trayectoria histórica (esto es, si existen entierros que correspondan a la ocupación de los jesuitas, es decir, hasta 1767), o bien, si dicho sector únicamente fue empleado con posterioridad a los jesuitas. En todo caso, es posible plantear como hipótesis que este sector fue reutilizado como espacio de inhumaciones hacia fines de siglo XIX y que estaría vinculado a la epidemia de cólera (cf. Teitelbaum 1998) que azotó al ámbito periurbano y rural tucumano durante el último cuarto de siglo XIX.

${ }^{10}$ Es posible que este valor remita a un comportamiento particular: o el valor de $122 \mathrm{mg} / \mathrm{kg}$ refleja una depositación diferencial producto del reentierro de restos óseos -en este sentido, Anastasio et al. (2005) registran un pequeño osario localizado al pie del actual campanario, por lo que se podría plantear que el valor de $\mathrm{A}^{\prime}$ estaría reflejando una situación particular de redepositación de restos óseos hacia fines de siglo XIX o inicios del XX-; o bien, la otra posibilidad, es que dicho valor constituya un indicio de la limitación de la técnica, aunque resulte complejo determinar cuál es el motivo del error.

${ }^{11}$ Uno de los co-autores del presente trabajo (V. A.), durante el período comprendido entre octubre de 2007 y diciembre de 2008, intervino como veedor judicial en las tareas de campo del Centro de Entrenamiento en Desminado Humanitario del Ejército Argentino y, posteriormente, y ya como perito del Juzgado Federal $\mathrm{N}^{\circ} 2$ (durante los meses de febrero y marzo de 2009, junto a R. Zurita), con el fin de detectar y registrar -a los fines de la reconstrucción histórica del CCD- una serie de caminos y sendas que comunicaban, durante el segundo quinquenio de la década de 1970, el lugar de reclusión con otras dependencias y con el acceso al predio militar por Ruta $\mathrm{N}^{\circ}$ 9. La relevancia de dicha instancia pericial radicaba en: determinar el camino original por donde los "grupos de tarea" (o "patotas") ingresaban los detenidos al espacio de reclusión clandestina y relevar aquellas modificaciones en el paisaje que remitían a la refuncionalización de una parte del predio militar como CCD (esto es, evidencias que contribuyan a dar cuenta de la conformación de un paisaje de exterminio en la ex Compañía). 


\section{Bibliografía citada}

Anastasio, G., V. H. Ataliva, M. L. Cohen, M. Di Pierro, S. M. L. López Campeny, C. Rivet, C. Somonte y S. Urquiza

2005 Análisis preliminar de los materiales recuperados en las "Ruinas de San José de Lules", Provincia de Tucumán. Actas del XIII Congreso Nacional de Arqueología Argentina 4: 133-142. Universidad Nacional de Córdoba, Córdoba [1999].

Ataliva, V.

2008 Arqueología, memorias y procesos de marcación social (acerca de las prácticas sociales posgenocidas en San Miguel de Tucumán). Notas de Investigación 1. GIAAT, Universidad Nacional de Tucumán, Tucumán - Editorial Mnemosyne, Buenos Aires.

Ataliva, V.

2010 En las entrañas del IAM... arqueología en el patio del Instituto (o una excusa para analizar la trayectoria de la arqueología en contextos históricos de Tucumán). Rastros en el camino... Trayectos e identidades de una Institución (comp. por P. Arenas, C. Aschero y C. Taboada), pp. 29-41. EDUNT, Tucumán.

Ataliva, V. H. y S. M. L. López Campeny

2002 Los problemas de circulación monetaria en el Tucumán Colonial del siglo XVIII a través del registro arqueológico. Las monedas recuperadas en la Estancia Jesuita de San José de Lules (Tucumán, República Argentina). Anales del Museo de América 10: 185-197.

Barba, L.

1990 Radiografía de un sitio arqueológico. Universidad Nacional Autónoma de México, México DF.

Barba, L. y S. Domínguez-Bella

2003 Crónica: 34th Internacional Symposium on Archaeometry. Revista Atlántica-Mediterránea de Prehistoria y Arqueología Social 6: 391-412.

Barba, L., R. Rodríguez y J. L. Córdova

1991 Manual de Técnicas Microquímicas de Campo para Arqueología. Universidad Nacional Autónoma de México, México DF.

Binder, P. V.

2008 Ingeniando Alternativas y Proyectando Técnicas. La prospección química como herramienta para la detección de inhumaciones clandestinas. Tesis de Grado inédita, Facultad de Ciencias Exactas y Tecnología, Universidad Nacional de Tucumán, Tucumán.

Binder, P. y V. Ataliva

2009 Prospección geoquímica e inhumaciones: la Estancia de San José de Lules como caso de estudio. Libro de las IX Jornadas de Comunicaciones de la Facultad de Ciencias Naturales e Instituto M. Lillo y II Interinstitucionales, Serie Monográfica y Didáctica 48, p. 103.

Boschetti, G., M. Díaz-Zorita, C. Quintero y M. Barraco

2003 Determinación del fósforo disponible en el suelo por el método de Bray. Informaciones Agronómicas del Cono Sur 17. 
Comisión Bicameral

1991 Informe de la Comisión Bicameral Investigadora de las Violaciones de los Derechos Humanos en la Provincia de Tucumán (1974-1983). Instituto de Estudios Políticos para América Latina y África, Tucumán.

\section{CONADEP}

1984 Nunca Más. Comisión Nacional Sobre la Desaparición de Personas - EUDEBA, Buenos Aires.

Cook, S. F. \& R. F. Heizer

1965 Studies on the chemical analysis of archaeological sites. University of California Press, Berkeley \& Los Angeles.

Díaz Vázquez, M.

1993 Aplicación de métodos fisicoquímicos en Arqueología. Espacio, Tiempo y Forma, Prehistoria y Arqueología 6: 249-262.

Eugenio, E. y A. Murgo

1994 Delimitación de un sitio arqueológico mediante el contenido de fósforo en el suelo. Arqueología 4: 199-211.

Eugenio, E. y A. Murgo

1996-98 Análisis de fosfatos en sitios arqueológicos mediante ensayos a la gota: principios, limitaciones y calibración semicuantitativa de la Técnica EIDIT. Palimpsesto 5: 24-40.

Feierstein, D.

2007 El genocidio como práctica social. Entre el nazismo y la experiencia argentina. Fondo de Cultura Económica, Buenos Aires.

García Azcárate, J., L. D. Vuoto y E. Ribotta

2002 Análisis preliminar del conjunto jesuítico San José de Lules. Actas del XIII Congreso Nacional de Arqueología Argentina 2: 127-139. Universidad Nacional de Córdoba, Córdoba [1999].

Hardin, P., J. Parnell y R. Terry

1999 Las comunidades rurales y los suelos de Piedras Negras, Petén. XII Simposio de Investigaciones Arqueológicas en Guatemala (ed. por J. P. Laporte y H. L. Escobedo), pp. 369-375. Museo Nacional de Arqueología y Etnología, Guatemala.

Jacobo, A. L., A. N. Vásquez y S. W. Solís

2004 Prospección química en excavaciones forenses en el Departamento de Quiché. XVII Simposio de Investigaciones Arqueológicas en Guatemala, 2003 (ed. por J. P. Laporte, B. Arroyo, H. L. Escobedo y H. Mejía), pp. 1050-1058. Museo Nacional de Arqueología y Etnología, Guatemala.

Leonardi, G., M. Miglavacca \& S. Nardi

1999 Soil Phosphorus Analysis as an Integrative Tool for Recognizing Buried Ancient Ploughsoils. Journal of Archaeological Science 26: 343-352. 
Ortiz, G.

2003 La aplicación de análisis químicos para la determinación de áreas de actividad en la región subandina de Jujuy. Cuadernos UNJu 20: 291-321.

Parnell, J. J., F. G. Fernández y R. E. Ferry

2002 Investigaciones químicas del suelo en Piedras Negras, Guatemala: Aplicaciones en arqueología. XV Simposio de Investigaciones Arqueológicas en Guatemala (ed. por J. P. Laporte, H. Escobedo y B. Arroyo), pp. 145-153. Museo Nacional de Arqueología y Etnología, Guatemala.

Ritz, K., L. Dawson \& D. Miller (eds.)

2009 Criminal and Environmental Soil Forensics. Springer, New York.

Rodríguez Suárez, R.

2006 Arqueología de una búsqueda; una búsqueda arqueológica. La historia del hallazgo de los restos del Che Guevara. Arqueología de la represión y la resistencia en América Latina (1960-1980) (comp. por P. P. Funari y A. Zarankin), pp. 17-37. Editorial Brujas, Córdoba.

Roldán, J.

2004 Efectos antrópicos del uso del suelo durante el Formativo en el Valle de Tafí. Tesis de Grado inédita. Universidad Nacional de Tucumán, Tucumán.

Roldán, J.

2009 La detección de actividad humana pasada a través de técnicas químicas. Revista Arakuku 1: 16-29.

Roldán, J., M. M. Sampietro Vattuone, L. Neder y M. A. Vattuone

2008 Efectos antrópicos de uso de suelos durante el formativo en el Valle de Tafí (Tucumán - Argentina). Chungara 40(2): 161-172.

Sampietro Vattuone, M. M.

2002 Contribución al conocimiento geoarqueológico del Valle de Tafí, Tucumán (Argentina). Tesis Doctoral inédita. Universidad Nacional de Tucumán, Tucumán.

Somigliana, M. y D. Olmo

2002 Qué significa identificar. Encrucijadas 15: 22-35.

Teitelbaum, V.

1998 La prédica higienista en la construcción de una imagen de la maternidad en Tucumán, Argentina, a fines del siglo XIX y comienzos del XX. Papeles de Población 16: 185-200.

Terry, R. E., S. Nelson, J. Carr, J. Parnell, P. Hardin, M. Jackson \& S. Houston 2000 Quantitative phosphorus measurement: a field test procedure for archaeological site análisis at Piedras Negras, Guatemala. Geoarchaelogy 15: 151-166.

Zalba, P., O. Bravo, N. M. Amiotti y N. Peinemann

2002 Métodos alternativos para determinar las disponibilidades de fósforo en suelos agrícolas. Ciencia del Suelo 20(1): 50-53. 